\title{
Image reconstruction/synthesis from nonuniform data and zero/threshold crossings
}

\author{
Yongwan Park \\ Korea Mobile Telecommunication \\ Central Research Center \\ System Technology, CDMA Task Force \\ Taejon, Korea 305-348 \\ and \\ California Institute of Technology \\ Department of Electrical Engineering \\ Pasadena, California 91125 \\ E-mail: ypark@kmnms4.kmt.re.kr \\ Mehrdad Soumekh \\ State University of New York at Buffalo \\ Department of Electrical \& Computer \\ Engineering \\ Amherst, New York 14260
}

\begin{abstract}
We address the problem of reconstructing functions from their nonuniform data and zero/threshold crossings. We introduce a deterministic process via the Gram-Schmidt orthonormalization procedure to reconstruct functions from their nonuniform data and zero/threshold crossings. This is achieved by first introducing the nonorthogonal basis functions in a chosen 2-D domain (e.g., for a band-limited signal, a possible choice is the 2-D Fourier domain of the image) that span the signal subspace of the nonuniform data. We then use the Gram-Schmidt procedure to construct a set of orthogonal basis functions that span the linear signal subspace defined by the nonorthogonal basis functions. Next, we project the $N$-dimensional measurement vector ( $N$ is the number of nonuniform data or threshold crossings) onto the newly constructed orthogonal basis functions. Finally, the function at any point can be reconstructed by projecting the representation with respect to the newly constructed orthonormal basis functions onto the reconstruction basis functions that span the signal subspace of the evenly spaced sampled data. The reconstructed signal gives the minimum mean square error estimate of the original signal. This procedure gives error-free reconstruction provided that the nonorthogonal basis functions that span the signal subspace of the nonuniform data form a complete set in the signal subspace of the original band-limited signal. We apply this algorithm to reconstruct functions from their unevenly spaced sampled data and zero crossings and also apply it to solve the problem of synthesis of a 2-D band-limited function with the prescribed level crossings.
\end{abstract}

Subject terms: digital image recovery and synthesis, nonharmonic Fourier function; image reconstruction; nonuniform sampling; Gram-Schmidt orthogonalization; zero crossings; band-limited functions.

Optical Engineering 33(10), 3290-3301 (October 1994).

\section{Introduction}

We consider the problem of reconstructing functions from their unevenly spaced sampled data and zero/threshold crossings. Reconstruction from the finite unevenly spaced sampled data has a variety of applications in many areas. ${ }^{1-3}$ The problem of reconstructing a 2-D signal from its nonuniform data arises in certain medical imaging problems where either the measurement domain is nonuniform or the measured data are translated to nonuniform samples of the desired image. ${ }^{4,5}$ In radio astronomy, the irregular data that are obtained from an aperture synthesis radio telescope are applied to the reconstruction of 2-D functions. ${ }^{6}$ Reconstructing a signal from jittered samples occurs in speech processing because those samples are common due to fluctuations of tape recorder speed or channel delay distortion. ${ }^{1}$

A special case of signal reconstruction from unevenly spaced samples is signal reconstruction from zero/threshold crossings. Zero crossing is important information in communication when the signal is clipped or distorted during translation and/or only zero-crossing information is available.

Paper DIR-09 received Nov. 18, 1993; revised manuscript received July 10, 1994; accepted for publication July 25, 1994.

(C) 1994 Society of Photo-Optical Instrumentation Engineers. 0091-3286/94/\$6.00
A band-limited low-pass function may be uniquely represented as the entire function by its zero crossings (real and complex zeros). ${ }^{7}$ Since it is difficult to determine complex zeros in practice, the real-zero conversion method is used to convert complex zeros into real zeros. ${ }^{8}$ Thus the converted band-limited signal can be represented only with the real zeros. Kay and Sudhaker ${ }^{9}$ analyzed the spectrum of the signal and reconstructed the signal using information of zero crossing. Reconstruction from threshold crossing significantly reduces the size of the database (image compression) required to store medical images, e.g., $x$-ray images. Threshold crossings can also be used to solve the problem of synthesis of 2-D band-limited functions. This problem occurs when we want to generate a prescribed binary image at the output of a diffraction-limited imaging system with a high-contrast recording device, e.g., a microlithographic camera or laser printer. $^{10-12}$

Many reconstruction methods from nonuniform samples have been proposed since Shannon's sampling theorem was introduced. Shannon's sampling theorem states that any band-limited signal can be recovered exactly by low-pass filtering of its infinitely uniform samples, provided that the sampling rate is greater than twice the highest frequency of the band-limited signal. Because of the distinctive two rea- 
sons- the finite number of sampled data and the unevenly spaced sampled data-we cannot apply Shannon's sampling theorem for the exact reconstruction of a band-limited signal. The folk theorem states that if the average sampling rate is higher than the Nyquist rate, irrespective of the sampling set (unevenly spaced or jittered), the error-free reconstruction of a band-limited signal is possible. Beutler ${ }^{13}$ proved that the error-free reconstruction can be achieved provided that the basis functions, $\left\{\exp \left(i \omega t_{n}\right)\right\}$, form a complete set in the signal subspace of the original band-limited signal. Higgins ${ }^{14}$ expressed the band-limited signal by interpolating unevenly spaced sampled data. The interpolating function, which is a biorthogonal of the sinc function, can be represented as the Lagrange interpolation function provided that evenly spaced sampled points are jittered at less than $25 \%$ of the Nyquist rate. Chen and Allebach ${ }^{15}$ estimated a band-limited function from unevenly spaced sampled data by interpolating unevenly spaced sampled points with the coefficients based on the minimum mean square error (MMSE) criterion. Their interpolating kernel is the inverse Fourier transform of the normalized indicator function. The coefficients are obtained via inversion of the point set matrix, which is determined by a set of sampling points and unevenly spaced sampled values. This estimator is identical to Yen's, ${ }^{16}$ which is based on the minimum energy of the signal.

In this paper, we introduce a deterministic signal subspace process via the Gram-Schmidt orthogonalization procedure to reconstruct functions from their unevenly spaced sampled data and zero/threshold crossings. The reconstructed signal gives the MMSE estimate of the original signal. This procedure gives error-free reconstruction provided that the nonorthogonal basis functions that span the signal subspace of the nonuniform data form a complete set in the signal subspace of the original band-limited signal. Finally, we apply this algorithm to reconstruct functions from their unevenly spaced sampled data and zero crossings and also apply it to solve the problem of synthesis of a 2-D band-limited function with the prescribed level crossings.

\section{Reconstruction Algorithm}

\subsection{Formulation of the Algorithm}

We present a deterministic process via the Gram-Schmidt orthonormalization procedure to reconstruct functions from their nonuniform data. This can be achieved by introducing the nonorthogonal basis functions in a chosen 1- or 2-D domain that span the signal subspace of the nonuniform data. We first introduce the nonorthogonal basis functions $\left\{\bar{u}_{k} ; k=1,2, \ldots, N\right\}$ that span the signal subspace of the available nonuniform data. As a special case, the nonharmonic Fourier functions are used as the nonorthogonal basis functions to reconstruct band-limited functions from their nonuniform samples in Sec. 3. The general form of nonorthogonal basis functions can be expressed in the form of a matrix, i.e.,

$$
\mathbf{U}=\left[\begin{array}{c}
\bar{u}_{1} \\
\bar{u}_{2} \\
\vdots \\
\bar{u}_{N}
\end{array}\right]=\left[\begin{array}{cccc}
u_{t_{1}}\left(\omega_{0}\right) & u_{t_{1}}\left(2 \omega_{0}\right) & \ldots & u_{t_{1}}\left(N \omega_{0}\right) \\
u_{t_{2}}\left(\omega_{0}\right) & u_{t_{2}}\left(2 \omega_{0}\right) & \ldots & u_{t_{2}}\left(N \omega_{0}\right) \\
\vdots & \vdots & \ldots & \vdots \\
u_{t_{N}}\left(\omega_{0}\right) & u_{t_{N}}\left(2 \omega_{0}\right) & \ldots & u_{t_{N}}\left(N \omega_{0}\right)
\end{array}\right]
$$

where $\omega_{0}$ is the fundamental frequency and $\left\{t_{n} ; n=1,2\right.$, $\ldots, N\}$ are the nonuniform sampling points of the signal. Note that the matrix $\mathbf{U}$ is a linearly independent sequence, provided that the nonuniform samples are distinct.

We then use the Gram-Schmidt orthonormalization procedure to construct a set of orthonormal basis functions from the previously mentioned nonorthogonal basis functions. Let $\left\{\bar{\phi}_{k} ; k=1,2, \ldots, N\right\}$ be orthonormal basis functions that are obtained by the Gram-Schmidt orthonormalization procedure. The orthonormal basis functions in a matrix form are

$$
\Phi=\left[\begin{array}{c}
\bar{\phi}_{1} \\
\bar{\phi}_{2} \\
\vdots \\
\bar{\phi}_{N}
\end{array}\right]=\left[\begin{array}{cccc}
\phi_{t_{1}}\left(\omega_{0}\right) & \phi_{t_{1}}\left(2 \omega_{0}\right) & \ldots & \phi_{t_{1}}\left(N \omega_{0}\right) \\
\phi_{t_{2}}\left(\omega_{0}\right) & \phi_{t_{2}}\left(2 \omega_{0}\right) & \ldots & \phi_{t_{2}}\left(N \omega_{0}\right) \\
\vdots & \vdots & \ldots & \vdots \\
\phi_{t_{N}}\left(\omega_{0}\right) & \phi_{t_{N}}\left(2 \omega_{0}\right) & \ldots & \phi_{t_{N}}\left(N \omega_{0}\right)
\end{array}\right]
$$

We now investigate the problem of finding the best estimate of the desired signal in the signal subspace spanned by the orthonormal basis functions $\left\{\bar{\phi}_{k}\right\}$. Let $\mathbf{F}=[F(1), F(2), \ldots$, $F(N)]$ be the spectrum of the original signal and $\hat{\mathbf{F}}$ be its estimate. The MMSE estimate of the spectrum $\mathbf{F}$ can be represented by coefficients $\left\{\alpha_{k}\right\}$ with respect to the basis functions $\left\{\bar{\phi}_{k}\right\}$, which is

$\hat{\mathbf{F}}=\sum_{k=1}^{N} \alpha_{k} \bar{\phi}_{k}$

When the basis functions $\left\{\bar{\phi}_{k}\right\}$ form a complete orthonormal set, the spectrum $\mathbf{F}$ can be represented exactly using those basis functions with scalars $\left\{\alpha_{k}\right\}$, i.e.,

$\mathbf{F}=\sum_{k=1}^{N} \alpha_{k} \bar{\phi}_{k}$

In Fig. 1, the spectrum $\mathbf{F}$ is expressed with $\left\{\alpha_{k}\right\}$ and the orthonormal basis functions $\left\{\bar{\phi}_{k}\right\}$, which are constructed by the $\left\{\bar{u}_{k}\right\}$ and nonuniform sample $f_{k}$ obtained by projecting $\mathbf{F}$

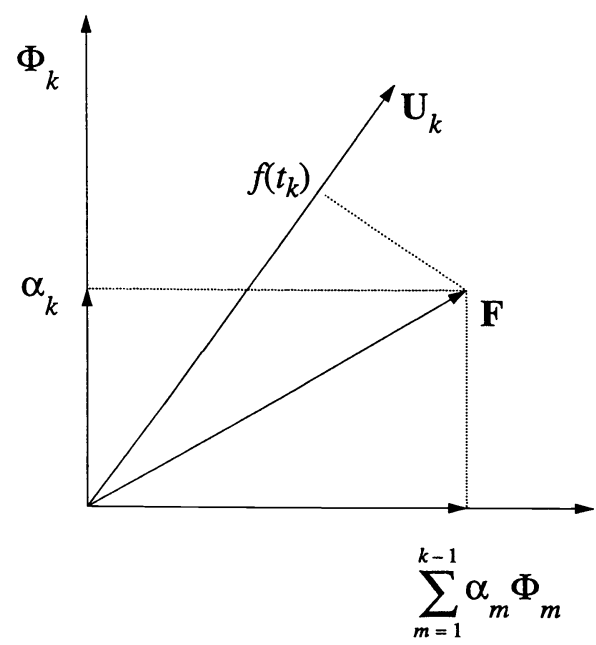

Fig. 1 The relations among spectrum, basis functions, coefficient, and nonuniform sample. 
onto $\bar{u}_{k}$. The detailed procedure for obtaining the nonuniform sample $f_{k}$, which can be obtained by projecting $\mathbf{F}$ onto $\bar{u}_{k}$, is given in the next section.

The coefficients $\left\{\alpha_{k}\right\}$, which give the MMSE estimate of the original signal, can be found by projecting the $N$ dimensional measurement vector $\mathbf{F}$ onto the orthonormal basis functions $\Phi$. According to the projection theorem, the best coefficients $\left\{\alpha_{k}\right\}$ in the MMSE sense can also be determined by requiring that the error between $\mathbf{F}$ and $\hat{\mathbf{F}}$ be orthogonal to every $\left\{\bar{u}_{i}\right\}$, i.e.,

$\left\langle\mathbf{F}-\hat{\mathbf{F}}, \bar{u}_{i}\right\rangle=0, \quad$ for $i=1,2, \ldots, N$.

Note that $\langle\cdot, \cdot\rangle$ denote the inner product. By linearity of the inner product, Eq. (5) can be rewritten as

$\left\langle\mathbf{F}, \bar{u}_{i}\right\rangle=\left\langle\hat{\mathbf{F}}, \bar{u}_{i}\right\rangle, \quad$ for $i=1,2, \ldots, N$.

By substitution of Eq. (3) into Eq. (6), we can rewrite Eq. (6) using the definitions of inner product and linear combination as follows:

$$
\begin{aligned}
\left\langle\mathbf{F}, \bar{u}_{i}\right\rangle= & \left\langle\sum_{k=1}^{N} \alpha_{k} \bar{\phi}_{k}, \bar{u}_{i}\right\rangle \\
= & \alpha_{1}\left\langle\bar{\phi}_{1}, \bar{u}_{i}\right\rangle+\alpha_{2}\left\langle\bar{\phi}_{2}, \bar{u}_{i}\right\rangle+\ldots \\
& +\alpha_{N}\left\langle\bar{\phi}_{N}, \bar{u}_{i}\right\rangle, \quad \text { for } i=1,2, \ldots, N .
\end{aligned}
$$

Generally, the nonorthogonal basis functions can be expressed as a linear combination of their orthonormal basis functions based on the property of the Gram-Schmidt orthonormalization procedure, i.e.,

$\bar{u}_{j}=\sum_{i=1}^{j} b_{j i} \bar{\phi}_{i}$

where $b_{j i}$ is the inner product of $\bar{u}_{j}$ and $\bar{\phi}_{i}$. Note that

$$
\left\langle\bar{\phi}_{k}, \bar{u}_{j}\right\rangle=0 \text {, if } j<k \text {. }
$$

Consider Eq. (7) for any $\bar{u}_{j}$ (the $j$ 'th nonorthogonal basis vector) with Eq. (9), i.e.,

$\left\langle\mathbf{F}, \bar{u}_{j}\right\rangle=\alpha_{j}\left\langle\bar{\phi}_{j}, \bar{u}_{j}\right\rangle+\sum_{k=1}^{j-1} \alpha_{k}\left\langle\bar{\phi}_{k}, \bar{u}_{j}\right\rangle$.

Hence,

$\alpha_{j}=\frac{\left\langle\mathbf{F}, \bar{u}_{j}\right\rangle-\sum_{k=1}^{j-1} \alpha_{k}\left\langle\bar{\phi}_{k}, \bar{u}_{j}\right\rangle}{\left\langle\bar{\phi}_{j}, \bar{u}_{j}\right\rangle}$, for $j=2,3, \ldots, N$

and the initial basis and coefficient are

$\left\{\begin{array}{l}\bar{\phi}_{1}=\bar{u}_{1} /\left\|\bar{u}_{1}\right\| \\ \alpha_{1}=\left\langle\mathbf{F}, \bar{u}_{1}\right\rangle /\left\langle\bar{\phi}_{1}, \bar{u}_{1}\right\rangle .\end{array}\right.$

Let $\left\{\bar{\psi}_{k} ; k=1,2, \ldots, N\right\}$ be the orthonormal basis functions on which we desire to reconstruct a function. The basis functions $\Psi$ can be expressed in the matrix form

$$
\Psi=\left[\begin{array}{c}
\bar{\psi}_{1} \\
\bar{\psi}_{2} \\
\vdots \\
\bar{\psi}_{N}
\end{array}\right]=\left[\begin{array}{cccc}
\psi_{t_{1}}\left(\omega_{0}\right) & \psi_{t_{1}}\left(2 \omega_{0}\right) & \ldots & \psi_{t_{1}}\left(N \omega_{0}\right) \\
\psi_{t_{2}}\left(\omega_{0}\right) & \psi_{t_{2}}\left(2 \omega_{0}\right) & \ldots & \psi_{t_{2}}\left(N \omega_{0}\right) \\
\vdots & \vdots & \ldots & \vdots \\
\psi_{t_{N}}\left(\omega_{0}\right) & \psi_{t_{N}}\left(2 \omega_{0}\right) & \ldots & \psi_{t_{N}}\left(N \omega_{0}\right)
\end{array}\right]
$$

We thus express the function $\hat{\mathbf{F}}$, which spans the other orthonormal basis $\left\{\bar{\psi}_{k}\right\}$, with scalar $\left\{\beta_{k}\right\}$ as

$\hat{\mathbf{F}}=\sum_{k=1}^{N} \beta_{k} \bar{\psi}_{k}$

The function at any point can be reconstructed by projecting the representation $\left\{\alpha_{k} \bar{\phi}_{k}\right\}$ with respect to the newly constructed orthonormal basis functions onto the reconstruction basis functions $\left\{\bar{\psi}_{k}\right\}$, i.e.,

$$
\begin{aligned}
\beta_{m} & =\left\langle\hat{\mathbf{F}}, \bar{\psi}_{m}\right\rangle \\
& =\sum_{k=1}^{N} \alpha_{k}\left\langle\bar{\phi}_{k}, \bar{\psi}_{m}\right\rangle, \text { for any } m .
\end{aligned}
$$

As the extension of the 1-D reconstruction, the nonorthogonal basis functions $\mathbf{W}_{\mathbf{I}}$, which span the 2-D signal subspace of the nonuniform data, are introduced and can be expressed in a matrix form as follows:

$\mathbf{W}_{\mathbf{I}}=\left[\begin{array}{c}\bar{w}_{I_{1}} \\ \bar{w}_{I_{2}} \\ \vdots \\ \bar{w}_{I_{K}}\end{array}\right]=\left[\begin{array}{cccc}u_{x_{1}}\left(\omega_{0}\right) \mathbf{v}_{y_{1}} & u_{x_{1}}\left(2 \omega_{0}\right) \mathbf{v}_{y_{1}} & \ldots & u_{x_{1}}\left(M \omega_{0}\right) \mathbf{v}_{y_{1}} \\ u_{x_{2}}\left(\omega_{0}\right) \mathbf{v}_{y_{1}} & u_{x_{2}}\left(2 \omega_{0}\right) \mathbf{v}_{y_{1}} & \ldots & u_{x_{2}}\left(M \omega_{0}\right) \mathbf{v}_{y_{1}} \\ \vdots & \vdots & \ldots & \vdots \\ u_{x_{M}}\left(\omega_{0}\right) \mathbf{v}_{y_{N}} & u_{x_{M}}\left(2 \omega_{0}\right) \mathbf{v}_{y_{N}} \ldots & u_{x_{M}}\left(M \omega_{0}\right) \mathbf{v}_{y_{N}}\end{array}\right]$,

where $K=M N, \mathbf{v}_{y_{j}}=\left[v_{y_{j}}\left(\omega_{0}\right), v_{y_{j}}\left(2 \omega_{0}\right), \ldots, v_{y_{j}}\left(N \omega_{0}\right)\right]$, and $\left\{x_{m}, y_{n}\right\}$ are the nonuniform sampling points of the image. The Gram-Schmidt orthonormalization procedure is used to construct the orthonormal basis functions $\Gamma_{\mathbf{I}}=\left[\bar{\gamma}_{I_{1}}\right.$, $\left.\bar{\gamma}_{I_{2}}, \ldots, \bar{\gamma}_{I_{K}}\right]^{T}$ that span the 2-D signal subspace defined by the previously mentioned nonorthogonal basis functions.

The MMSE estimate of a 2-D function $\hat{\mathbf{F}}_{\mathbf{I}}$ can be represented by the coefficients $\left\{\alpha_{I_{k}}\right\}$ with respect to the orthonormal basis functions $\left\{\bar{\gamma}_{I_{k}}\right\}$, i.e.,

$\hat{\mathbf{F}}_{\mathbf{I}}=\sum_{k=1}^{K} \alpha_{I_{k}} \bar{\gamma}_{I_{k}}$

The coefficients $\left\{\alpha_{I}\right\}$, which give the MMSE estimate of the original image, can be found in the same way in the 1-D case, i.e.,

$\alpha_{I_{j}}=\frac{\left\langle\mathbf{F}_{\mathbf{I}}, \bar{w}_{I_{j}}\right\rangle-\sum_{k=1}^{j-1} \alpha_{I_{k}}\left\langle\bar{\gamma}_{I_{k}}, \bar{w}_{I_{j}}\right\rangle}{\left\langle\bar{\gamma}_{I_{j}}, \bar{w}_{I_{j}}\right\rangle}$.

The desired images can be expressed with respect to the orthonormal reconstruction basis $\Theta_{\mathbf{I}}$ as 
$\beta_{I_{m}}=\sum_{k=1}^{K} \alpha_{I_{k}}\left\langle\bar{\gamma}_{I_{k}}, \bar{\theta}_{I_{m}}\right\rangle, \quad$ for any $m$

\subsection{The Completeness of the Basis Function}

It is shown that the sampling points that are jittered less than $25 \%$ of the Nyquist rate from the evenly spaced sampled points give the sufficient condition for the basis functions $\left\{\exp \left(i \omega t_{n}\right)\right\}$ to form a complete set. ${ }^{13}$ When the basis functions form a complete set, an error-free estimate of the signal is possible. For example, according to the projection theory, it is true that

$$
\langle\mathbf{F}-\hat{\mathbf{F}}, \mathbf{U}\rangle=0 \text {. }
$$

If the basis functions $\mathbf{U}$ in Eq. (19) form a complete set, there is no function orthogonal to $\mathbf{U}$ except the null function. Thus the error between the original and the estimated function should be zero, i.e., $\mathbf{F}-\hat{\mathbf{F}}=0$.

If we can represent $\Psi$ as a linear combination of $\left\{\bar{u}_{k}\right\}$, the basis functions $\mathbf{U}$ form a complete set. Because we cannot estimate $\Psi$ directly from $\mathbf{U}$, we introduce the orthonormal basis $\Phi$. We thus estimate the basis functions $\Psi$ by projecting $\Psi$ onto the orthonormal basis $\Phi$. The necessary and sufficient condition for the basis $\Phi$ to be a complete set is given by the following basis error:

$\lim _{N \rightarrow \infty}\left\|\Psi-\sum_{k=1}^{N}\left\langle\Psi, \bar{\phi}_{k}\right\rangle \bar{\phi}_{k}\right\|=0$

Note that $\Phi$ and $\mathbf{U}$ are the same signal subspace. Thus the completeness of basis $\mathbf{U}$ can be determined by the basis error of Eq. (20).

\section{Applications of Our Algorithm}

In this section, we apply our algorithm to the reconstruction of periodic band-limited functions from their unevenly spaced samples and zero crossings. Bandpass signals are also reconstructed without modulation. We also apply our algorithm to solve the problem of synthesis of 2-D band-limited functions with the prescribed threshold crossings.

Consider the signal $f_{l}(t)$, which is periodic with period $T=2 \pi / \omega_{0}$ and possesses no harmonics above the $N$ 'th. Let $B$ be the single-sided bandwidth in hertz. The real periodic signal $f_{l}(t)$ can be expressed in terms of the trigonometric polynomials, that is,

$f_{l}(t)=\sum_{k=-N}^{N} a_{k} \exp \left(i k \omega_{0} t\right)$

where

$a_{-k}=a_{k}^{*} \quad$ and $\quad N=\frac{2 \pi B}{\omega_{0}}=B T$.

The $2 N+1$ Fourier coefficients $\left\{a_{k}\right\}$ can be completely determined by a minimum of $2 N+1$ distinct values of $f_{l}(t)$. If the given distinct values are the evenly spaced samples with a sample spacing of $\Delta_{t}=T /(2 N+1)$, then the sampling expansion for the harmonic-limited periodic signal can be represented as follows:
$f_{l}(t)=\frac{1}{M} \sum_{n=1}^{M} f_{l}\left(n \Delta_{t}\right) \frac{\sin \frac{M}{2} \omega_{0}\left(t-n \Delta_{t}\right)}{\sin \frac{\omega_{0}}{2}\left(t-n \Delta_{t}\right)}$,

where $M=2 N+1$.

\subsection{Reconstruction from Nonuniform Data}

\subsubsection{Reconstruction of low-pass signals}

Let $f_{l}\left(t_{n}\right)$ be the nonuniform sampling value that is sampled at $\left\{t=t_{n} ; n=1,2, \ldots, M\right\}$, where $t_{n}=t_{n-M}+T$, i.e., $M$ locations within a time period of $T$ and those locations are repeated every $T$.

We introduce the nonorthogonal basis functions $\mathbf{U}_{\mathbf{L}}$ that span the signal subspace of the nonuniform data as the nonharmonic sinusoidal functions, i.e.,

$\mathbf{U}_{\mathbf{L}}=\left[\begin{array}{cccc}\exp \left[i(-N) \omega_{0} t_{1}\right] & \exp \left[i(-N+1) \omega_{0} t_{1}\right] & \ldots & \exp \left[i(N) \omega_{0} t_{1}\right] \\ \exp \left[i(-N) \omega_{0} t_{2}\right] & \exp \left[i(-N+1) \omega_{0} t_{2}\right] & \ldots & \exp \left[i(N) \omega_{0} t_{2}\right] \\ \vdots & \vdots & \ldots & \vdots \\ \exp \left[i(-N) \omega_{0} t_{M}\right] & \exp \left[i(-N+1) \omega_{0} t_{M}\right] & \ldots & \exp \left[i(N) \omega_{0} t_{M}\right]\end{array}\right]$

All vectors in $\mathbf{U}_{\mathbf{L}}$ are linearly independent provided that the sampling points $\left\{t_{n} ; n=1,2, \ldots, M\right\}$ are distinct.

We construct the orthonormal basis function $\phi_{\mathbf{L}}=\left[\bar{\phi}_{l}\right.$, $\left.\bar{\phi}_{l_{2}}, \ldots, \bar{\phi}_{l_{\mathrm{M}}}\right]^{T}$ via the Gram-Schmidt orthonormalization procedure from $\mathbf{U}_{\mathbf{L}}=\left[\bar{u}_{l_{1}}, \bar{u}_{l_{2}}, \ldots, \bar{u}_{l_{M}}\right]^{T}$. We next evaluate the coefficients $\left\{\alpha_{l_{k}}\right\}$ with the basis functions mentioned earlier, i.e., the complex sinusoidal functions. The only term that must be determined for the coefficients is the first term of the numerator in Eq. (11), i.e., the inner product of the discrete Fourier transform (DFT) vector $\mathbf{F}_{\mathbf{L}}$ and the $j$ 'th unevenly spaced sampled vector $\bar{u}_{l_{j}}$. By definition of the inner product it can be expressed as follows:

$\left\langle\mathbf{F}_{\mathbf{L}}, \bar{u}_{l_{j}}\right\rangle=\sum_{k=-N}^{N} F_{l}(k) \exp \left(i k \omega_{0} t_{j}\right)$,

where $\mathbf{F}_{\mathbf{L}}=\left\{F_{l}(-N), F_{l}(-N+1), \ldots, F_{l}(0), \ldots, F_{l}(N-1)\right.$, $\left.F_{l}(N)\right]$. For all $\left\{\bar{u}_{l_{k}} ; k=1,2, \ldots, M\right\}$, the matrix representation of Eq. (24) is given by

$\left[\begin{array}{c}\left\langle\mathbf{F}_{\mathbf{L}}, \bar{u}_{l_{1}}\right\rangle \\ \left\langle\mathbf{F}_{\mathbf{L}}, \bar{u}_{l_{2}}\right\rangle \\ \vdots \\ \left\langle\mathbf{F}_{\mathbf{L}}, \bar{u}_{l_{M}}\right\rangle\end{array}\right]=\mathbf{U}_{\mathbf{L}} \cdot\left[\begin{array}{c}F_{l}(-N) \\ F_{l}(-N+1) \\ \vdots \\ F_{l}(N)\end{array}\right]$.

With

$\frac{\sin \frac{(2 N+1)}{2}\left(\omega_{0} t\right)}{\sin \frac{1}{2}\left(\omega_{0} t\right)}=\sum_{k=-N}^{N} \exp \left(i k \omega_{0} t\right)$,

we can rewrite Eq. (22) as follows: 


$$
\begin{aligned}
f_{l}(t) & =\frac{1}{M} \sum_{k=-N}^{N} \sum_{n=1}^{M} f_{l}\left(n \Delta_{t}\right) \exp \left[i k\left(\omega_{0} t-2 \pi n / M\right)\right] \\
& =\frac{1}{M} \sum_{k=-N}^{N} F_{l}(k) \exp \left(i k \omega_{0} t\right)
\end{aligned}
$$

where $F_{l}(k)$, the DFT spectrum of $f(t)$, is

$F_{l}(k)=\sum_{n=1}^{M} f_{l}\left(n \Delta_{t}\right) \exp [-i(2 \pi / M) n k]$.

From Eq. (27), without loss of generality, the $j$ 'th nonuniform data can be represented by the DFT spectrum $\mathbf{F}_{\mathbf{L}}$, i.e.,

$f_{l}\left(t_{j}\right)=\frac{1}{M_{k}} \sum_{k=-N}^{N} F_{l}(k) \exp \left(i k \omega_{0} t_{j}\right)$.

Thus the nonuniform data can be related to the DFT spectrum as follows:

$$
\left[\begin{array}{c}
f_{l}\left(t_{1}\right) \\
f_{l}\left(t_{2}\right) \\
\vdots \\
f_{l}\left(t_{M}\right)
\end{array}\right]=\frac{1}{M} \mathbf{U}_{\mathbf{L}} \cdot\left[\begin{array}{c}
F_{l}(-N) \\
F_{l}(-N+1) \\
\vdots \\
F_{l}(N)
\end{array}\right] .
$$

With Eqs. (24) and (29), it is true that

$\left\langle\mathbf{F}_{\mathbf{L}}, \bar{u}_{l_{j}}\right\rangle=f_{l}\left(t_{j}\right) \cdot M$

By substitution of Eq. (31) into Eq. (11), the coefficients $\left\{\alpha_{l}\right\}$ can be written as the combinations of the nonuniform samples, basis functions, and their orthonormal basis functions, i.e.,

$\alpha_{l_{j}}=\frac{f_{l}\left(t_{j}\right) \cdot M-\sum_{k=1}^{j-1} \alpha_{l_{k}}\left\langle\bar{\phi}_{l_{k}}, \bar{u}_{l_{j}}\right\rangle}{\left\langle\bar{\phi}_{l_{j}}, \bar{u}_{l_{j}}\right\rangle}$.

Let $\Psi_{\mathbf{L}}$ be the set of complex sinusoidal functions on which we desire to reconstruct a low-pass signal, i.e.,

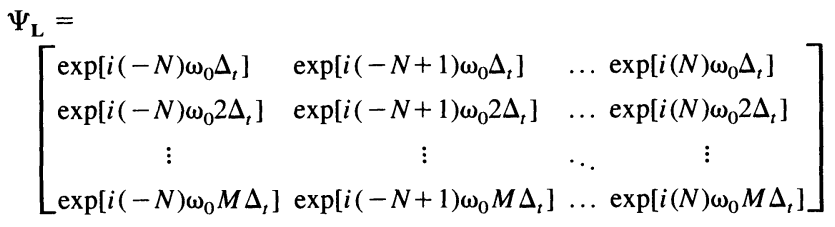

Since the set of orthogonal basis functions $\Psi_{\mathbf{L}}$ that span the signal subspace of nonuniform samples is defined as the harmonic complex sinusoidal functions, the projection of $\hat{\mathbf{F}}_{\mathbf{L}}$ onto the orthogonal basis function $\bar{\psi}_{l_{\mathrm{m}}}$ [see Eq.(14)] can be written by the DFT relationship with Eq. (29) as follows:

$$
\left\langle\hat{\mathbf{F}}_{\mathbf{L}}, \bar{\psi}_{l_{m}}\right\rangle=\hat{f}_{l}\left(m \Delta_{t}\right) \cdot M
$$

Therefore, with Eqs. (14) and (34) the desired signal can be expressed as $\hat{f}_{l}\left(m \Delta_{t}\right)=\frac{1}{M} \sum_{k=1}^{M} \alpha_{l_{k}}\left\langle\bar{\phi}_{l_{k}}, \bar{\psi}_{l_{m}}\right\rangle$

Experimental result. Two signals that are band-limited $[-5,5]$ and $[-16,16]$ are considered in the test. The 11 and 33 nonuniform samples were drawn from the random number generator in IMSL/LIB having uniform distribution in the interval $[-0.5,0.5]$, respectively. This assumes that each average sampling rate satisfies the Nyquist rate. The subroutine RNUN with ISEED $=123457$ was used to generate random points. We rescale the random points to be in the range of $[-1 / 2 B, 1 / 2 B]$. The unevenly spaced sampled points were obtained by the degrees of the deviation of the rescaled random points from the evenly spaced sampled points. We then wrapped the sampling points that are out of the interval within the interval. We first calculate the basis error to observe the completeness of the given basis functions $\mathbf{U}_{\mathbf{L}}$ using Eq. (20). Figure 2 depicts the error to signal power ratio (ESR) of the estimator for $\Psi_{L}$ depending on the degree of the deviation and randomly distributed samples for the 200 cases, respectively. The ESR is defined as

$\mathrm{ESR}=10 \log \frac{|o(t)-\hat{o}(t)|^{2}}{|o(t)|^{2}} \quad[\mathrm{~dB}]$,

where $o(t)$ and $\hat{o}(t)$ denote the original and estimated function, respectively. As shown in Fig. 2, the sampling sets that cause their basis errors to be below the line give the necessary and sufficient condition for their basis functions to form complete sets even though they violate Beutler's condition. ${ }^{13}$ We now reconstruct a signal with a set of samples that satisfy this condition. The tested signal that is band-limited in $[-5,5]$ is

$$
\begin{aligned}
f_{l}(t)= & \cos (2 \pi t)+0.2 \cos (4 \pi t-1) \\
& +1.2 \cos (6 \pi t-1.2)-0.7 \cos (10 \pi t+0.1) .
\end{aligned}
$$

Figure 3(a) shows the original signal with the randomly marked nonuniform samples. As expected, the reconstructed signal shown in Fig. 3(b) is identical to the original signal. The discrete Fourier spectra of the original and the reconstructed signal are compared in Table 1 . The reconstruction from the sampling sets that cause their basis errors to be less than $-200 \mathrm{~dB}$ produced a good estimation of signal by the numerical results. The average ESR of the signal, -89.01 $\mathrm{dB}$, and the basis functions, $-258.99 \mathrm{~dB}$, is obtained from the 200 sets of randomly distributed samples. We tested another band-limited signal that is band-limited $[-16,16]$ for the confirmation of our algorithm, i.e.,

$$
\begin{aligned}
y_{l}(t)= & y(t)+1.3 y(3 t-1.3)-0.9 y(13 t+0.7) \\
& +1.1 y(17 t+0.5)-2.4 y(32 t+0.4),
\end{aligned}
$$

where $y(t)=\cos (2 \pi t)$. Table 2 shows the average ESRs of the estimator $y_{l}(t)$ using the proposed algorithm and the matrix inversion method for 30 cases. Reconstruction of a periodic signal using the matrix inversion method is briefly discussed in Sec. 5.

\subsubsection{Reconstruction of bandpass signals}

For a bandpass signal produced by the amplitude modulation of a low-pass signal, the corresponding bandpass signal may 


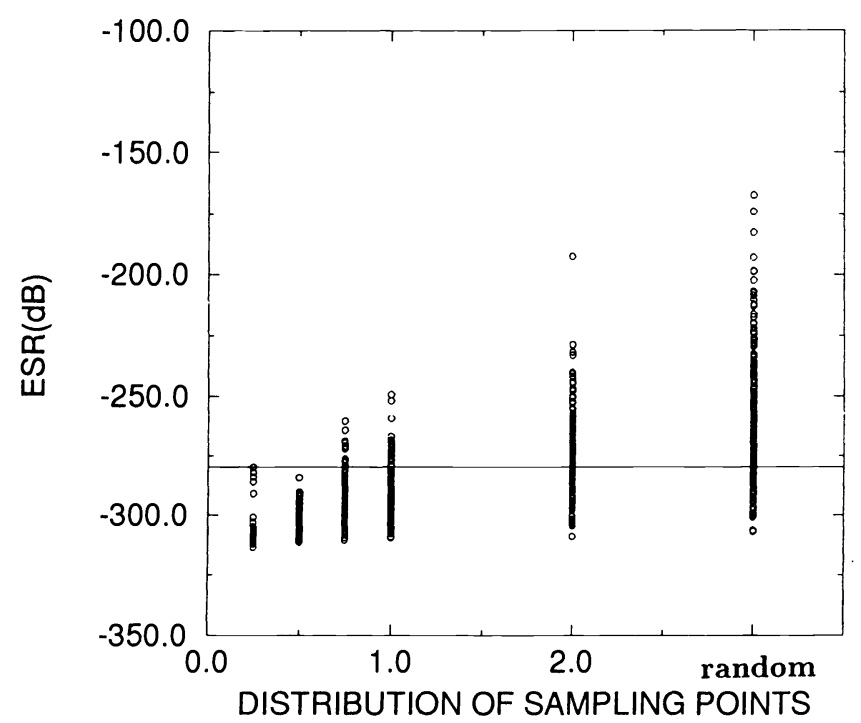

(a)

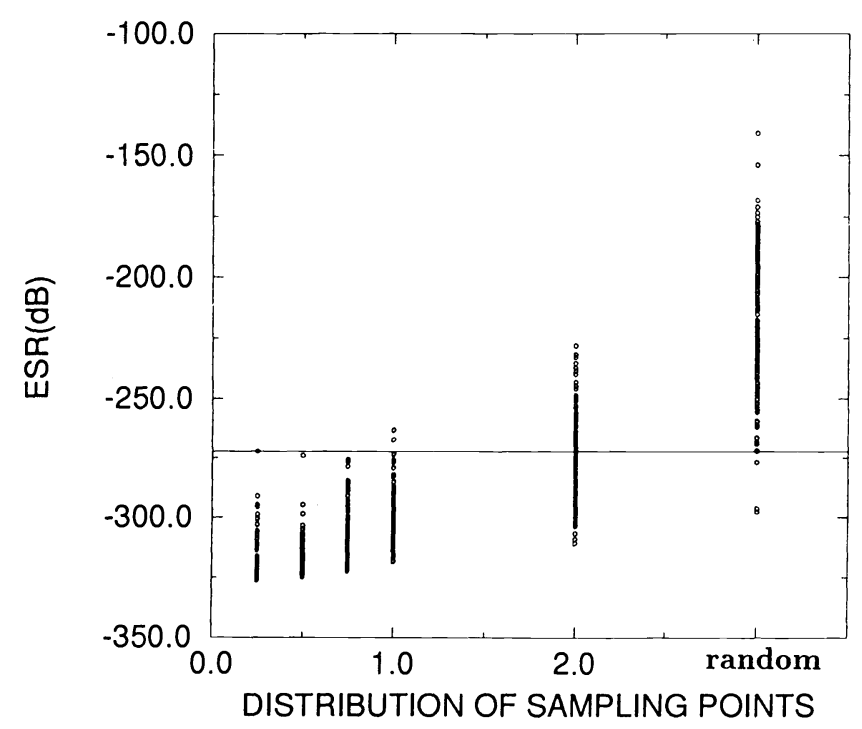

(b)

Fig. 2 The ESR of the estimator for $\Psi_{L}$ in decibels using the 200 sets of points (jittered and randomly distributed) whose bandwidths are $(a)[-5,5]$ and $(b)[-16,16]$.

be reconstructed from its samples that satisfy the Nyquist rate for the low-pass signal. Direct representation of a bandpass signal from uniformly spaced sampled data is introduced in Ref. 17.

Here, we introduce a direct reconstruction procedure for a bandpass signal from its unevenly spaced sampled data without modulation. Let $f_{p}(t)$ be the periodic bandpass signal whose frequency region is band-limited as

$\omega_{c}-\sigma / 2 \leqslant|\omega| \leqslant \omega_{c}+\sigma / 2$,

where $\left\{\omega_{c} ; \omega_{c} \geqslant \sigma / 2 \geqslant 0\right\}$ is the carrier frequency. The upper cutoff frequency, $\omega_{c}+\sigma / 2$, is an integer multiple of the fundamental frequency $\omega_{o}$, i.e., $k \omega_{0}$. In this case the carrier frequency is

$\omega_{c}=(k-N) \omega_{0}$.

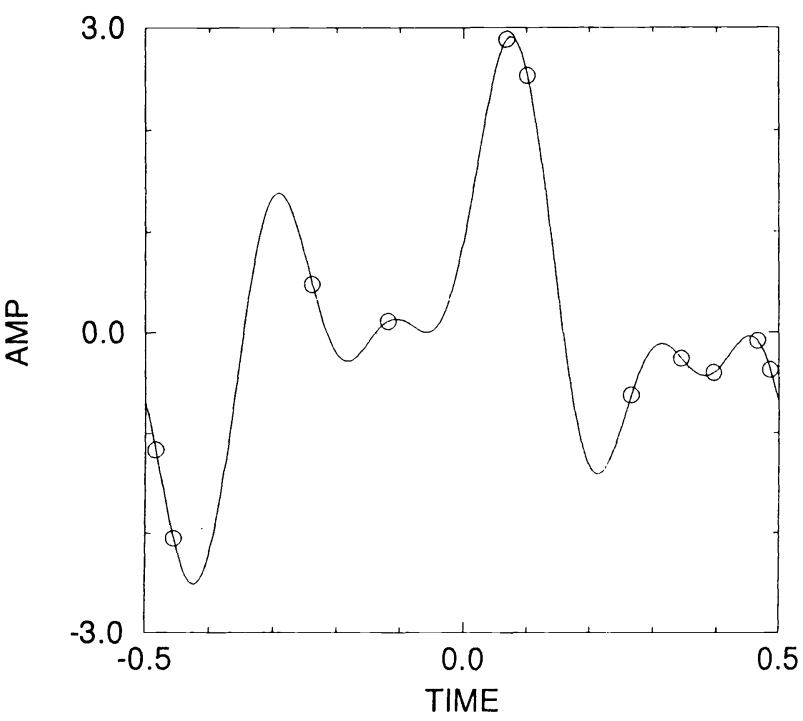

(a)

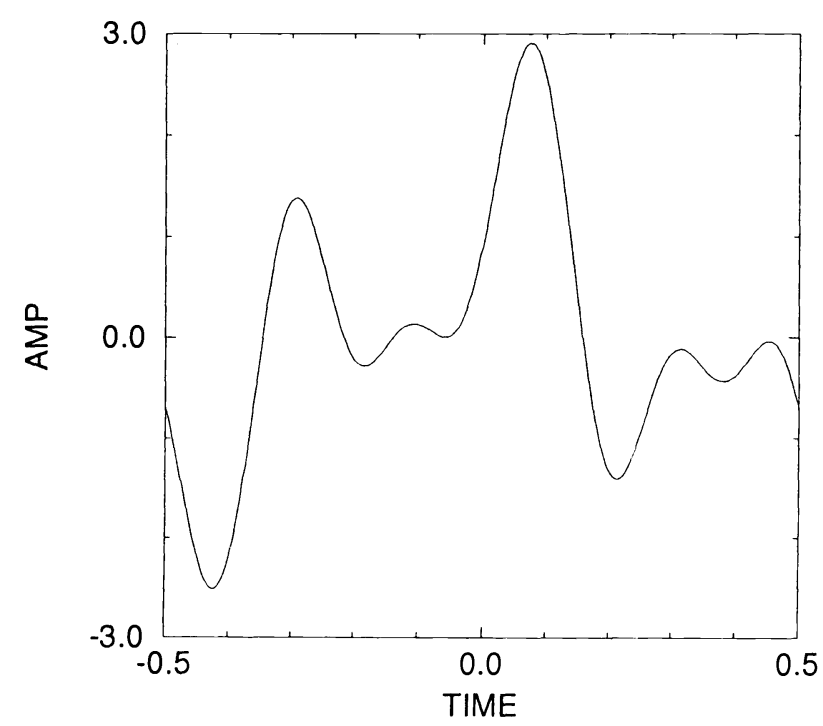

(b)

Fig. 3 (a) The original low-pass signal $f_{l}(t)$ with randomly marked nonuniform samples and (b) the reconstructed signal $\hat{f}_{/}(t)$.

Note that $k \geqslant N$. We introduce the nonorthogonal basis function $\mathbf{U}_{\mathbf{P}}$, which spans the bandpass signal subspace of the nonuniform data, i.e.,

$\mathbf{U}_{\mathbf{P}}=\left[\begin{array}{cccccc}u_{t_{1}}^{-k} & \ldots & u_{t_{1}}^{-(k-N)} u_{t_{1}}^{(k-N)} & \ldots & u_{t_{1}}^{k} \\ u_{t_{2}}^{-k} & \ldots & u_{t_{2}}^{-(k-N)} & u_{t_{2}}^{(k-N)} & \ldots & u_{t_{2}}^{k} \\ \vdots & \ldots & \vdots & \ldots & \vdots \\ u_{t_{J}}^{-k} & \ldots & u_{t_{J}}^{-(k-N)} & u_{t_{J}}^{(k-N)} & \ldots & u_{t_{J}}^{k}\end{array}\right]$

where $u_{t_{j}}^{N}=\exp \left(i N \omega_{0} t_{j}\right)$ and $J=2(N+1)$.

We first construct the orthonormal basis functions, $\Phi_{\mathbf{p}}=\left[\bar{\phi}_{p_{1}}, \bar{\phi}_{p_{2}}, \ldots, \bar{\phi}_{p_{J}}\right]^{T}$, via the Gram-Schmidt orthonormalization procedure from $\mathbf{U}_{\mathbf{P}}=\left[\bar{u}_{p_{1}}, \bar{u}_{p_{2}}, \ldots, \bar{u}_{p_{j}}\right]^{T}$. We next evaluate the coefficients $\left\{\alpha_{p}\right\}$ using Eq. (11). The first term 
Table 1 The DFT values from the original and estimated signal.

\begin{tabular}{l|l|l}
\hline DFT & THE ORIGINAL SIGNAL & THE ESTIMATED SIGNAL \\
\hline$F(-5)$ & $(-3.546085,1.499259)$ & $(-3.546096,1.499264)$ \\
\hline$F(-4)$ & $(5.389 E-08,-1.221 \mathrm{E}-07)$ & $(2.528 \mathrm{E}-05,2.856 \mathrm{E}-05)$ \\
\hline$F(-3)$ & $(2.391559,6.151459)$ & $(2.391593,6.151396)$ \\
\hline$F(-2)$ & $(0.594331,0.925618)$ & $(0.594239,0.925602)$ \\
\hline$F(-1)$ & $(5.500000,1.850 \mathrm{E}-07)$ & $(5.500000,-9.972 \mathrm{E}-05)$ \\
\hline$F(0)$ & $(1.385 \mathrm{E}-06,0.000 \mathrm{E}+00)$ & $(9.729 \mathrm{E}-05,-1.092 \mathrm{E}-14)$ \\
\hline$F(1)$ & $(5.500000,1.850 \mathrm{E}-07)$ & $(5.500000,-9.972 \mathrm{E}-05)$ \\
\hline$F(2)$ & $(0.594331,-0.925618)$ & $(0.594239,0.925602)$ \\
\hline$F(3)$ & $(2.391559,-6.151459)$ & $(2.391593,6.151396)$ \\
\hline$F(4)$ & $(5.389 \mathrm{E}-08,-1.221 \mathrm{E}-07)$ & $(2.528 \mathrm{E}-05,2.856 \mathrm{E}-05)$ \\
\hline$F(5)$ & $(-3.546085,-1.499259)$ & $(-3.546096,1.499264)$ \\
\hline
\end{tabular}

Table 2 Experimental results obtained from 30 sets of samples.

\begin{tabular}{c|c|c}
\hline $\begin{array}{c}\text { DISTRIBUTION } \\
\text { SAMPLES }\end{array}$ & $\begin{array}{c}\text { THE PROPOSED } \\
\text { METHOD (dB) }\end{array}$ & $\begin{array}{c}\text { THE INVERSION } \\
\text { METHOD (dB) }\end{array}$ \\
\hline $25 \%$ & -134.18 & -122.66 \\
\hline $50 \%$ & -130.33 & -113.25 \\
\hline $75 \%$ & -122.13 & -97.49 \\
\hline $100 \%$ & -119.34 & -81.95 \\
\hline $200 \%$ & -95.95 & -41.34 \\
\hline RANDOM & -37.17 & -15.56 \\
\hline
\end{tabular}

of the numerator in Eq. (11), $\left\langle\mathbf{F}_{\mathbf{P}}, \bar{u}_{p_{j}}\right\rangle$, can be rewritten as follows:

$$
\begin{aligned}
\left\langle\mathbf{F}_{\mathbf{P}}, \bar{u}_{p_{j}}\right\rangle= & \sum_{n=-k}^{-(k-N)} F_{p}(n) \exp \left(i n \omega_{0} t_{j}\right) \\
& +\sum_{n=k-N}^{k} F_{p}(n) \exp \left(i n \omega_{0} t_{j}\right),
\end{aligned}
$$

where $\mathbf{F}_{\mathbf{P}}=\left\{F_{p}(-k), \ldots, F_{p}[-(k-N)], F_{p}(k-N), \ldots\right.$, $\left.F_{p}(k)\right\}$

The nonuniform sampling data $\left\{f_{p}\left(t_{n}\right) ; n=1,2, \ldots, J\right\}$ can be related to $\mathbf{U}_{\mathbf{p}}$ and the DFT vector $\mathbf{F}_{\mathbf{p}}$ using the same procedure as in the case of the low-pass signal, i.e.,

$$
\left[\begin{array}{c}
f_{p}\left(t_{1}\right) \\
f_{p}\left(t_{2}\right) \\
\vdots \\
\vdots \\
f_{p}\left(t_{J}\right)
\end{array}\right]=\frac{1}{J} \mathbf{U}_{\mathbf{P}} \cdot\left[\begin{array}{c}
F_{p}(-k N) \\
\vdots \\
F_{p}[-(k-N)] \\
F_{p}(k-N) \\
\vdots \\
F_{p}(k N)
\end{array}\right]
$$

With Eqs. (42) and (43), it is true that

$\left\langle\mathbf{F}_{\mathbf{P}}, \bar{u}_{p_{j}}\right\rangle=f_{p}\left(t_{j}\right) \cdot J$.

Thus, the coefficients $\left\{\alpha_{p}\right\}$ can be expressed as

$$
\alpha_{p_{j}}=\frac{f_{p}\left(t_{j}\right) \cdot J-\sum_{k=1}^{j-1} \alpha_{p k},\left\langle\bar{\phi}_{p k}, \bar{u}_{p_{j}}\right\rangle}{\left\langle\bar{\phi}_{p_{j}}, \bar{u}_{p_{j}}\right\rangle} .
$$

Let $\Psi_{\mathbf{P}}$ be the complex sinusoidal functions on which we desire to reconstruct a bandpass signal,

$$
\Psi_{\mathbf{P}}=\left[\begin{array}{cccccc}
u_{\Delta_{t}}^{-k} & \ldots & u_{\Delta_{t}}^{-(k-N)} & u_{\Delta_{t}}^{(k-N)} & \ldots & u_{\Delta_{t}}^{k} \\
u_{2 \Delta_{t}}^{-k} & \ldots & u_{2 \Delta_{t}}^{-(k-N)} & u_{2 \Delta_{t}}^{(k-N)} & \ldots & u_{2 \Delta_{t}}^{k} \\
\vdots & \ldots & \vdots & \ldots & \vdots \\
u_{J \Delta_{t}}^{-k} & \ldots & u_{J \Delta_{t}}^{-(k-N)} & u_{J \Delta_{t}}^{(k-N)} & \ldots & u_{J \Delta_{t}}^{k}
\end{array}\right] .
$$

Hence, a bandpass signal can be represented as

$\hat{f}_{p}\left(m \Delta_{t}\right)=\frac{1}{J} \sum_{k=1}^{J} \alpha_{p_{k}}\left\langle\bar{\phi}_{p k}, \bar{\psi}_{p_{m}}\right\rangle$.

Experimental results. We used the periodic bandpass signal, which is band-limited $[-10,-5] \cup[5,10]$, as the original test signal, i.e.,

$$
\begin{aligned}
s_{p}(t)= & 0.2 \cos (10 \pi t-1)+1.2 \cos (16 \pi t-1.2) \\
& -0.7 \cos (20 \pi t+0.4) .
\end{aligned}
$$

The nonorthogonal basis functions $\mathbf{U}_{\mathbf{p}}$ have 12 spectral components in each vector. The 12 unevenly spaced sampled data were drawn in the interval $[-0.5,0.5]$. Compared with the conventional reconstruction method, our algorithm requires one more sample. The average ESRs of the signal, -101.82 $\mathrm{dB}$, and the basis functions, $-269.72 \mathrm{~dB}$, are obtained from 30 sets of randomly distributed samples.

\subsection{Reconstruction from Zero Crossings}

\subsubsection{Reconstruction of low-pass signals}

Consider the signal $x_{l}(t)$ that is band-limited $[-B, B]$ and has a combination of complex and real zeros. The $x_{l}(t)$ can be transformed to another band-limited signal $s_{l}(t)$, which has only real zeros by the following conversion technique. ${ }^{8}$ That is,

$s_{l}(t)=x_{l}(t)+A \sin (2 \pi f t)$,

where $f>B$ and $A>\max \left|x_{l}(t)\right|$.

Let $\left\{t_{k} ; k=1,2, \ldots, M-1\right\}$ be the real zero locations of $s_{l}(t)$, i.e.,

$s_{l}\left(t_{k}\right)=0$ for $k=1,2, \ldots, M-1$.

We choose any sampling point $\left\{t_{i} \neq t_{k} ; k=1,2, \ldots, M-1\right\}$ and put

$s_{l}\left(t_{M}\right)=s_{l}\left(t_{i}\right)$.

The available data can be related to the spectrum $\mathbf{S}_{\mathbf{L}}$ and the nonorthogonal basis functions $\mathbf{U}_{\mathbf{L}}$ as follows: 
$\left[\begin{array}{c}0 \\ 0 \\ \vdots \\ s_{l}\left(t_{M}\right)\end{array}\right]=\frac{1}{M} \mathbf{U}_{\mathrm{L}} \cdot\left[\begin{array}{c}S_{l}(-N) \\ S_{l}(-N+1) \\ \vdots \\ S_{l}(N)\end{array}\right]$.

Since $\left\{s_{l}\left(t_{k}\right) ; k=1,2, \ldots, M-1\right\}$ are zero, the coefficients $\left\{\alpha_{l_{k}} ; k=1,2, \ldots, M-1\right\}$ also become zero. Thus the only coefficient that we need to evaluate is $\alpha_{l_{M}}$. The coefficient $\alpha_{l_{M}}$ can be obtained from Eq. (11) and given by

$\alpha_{l_{M}}=\frac{s_{l}\left(t_{M}\right) \cdot M}{\left\langle\bar{\phi}_{l_{M}}, \bar{u}_{l_{M}}\right\rangle}$.

We then reconstruct $\hat{s}_{l}(t)$ by projecting only one component $\alpha_{l_{M}} \bar{\phi}_{l_{M}}$ onto the reconstruction basis $\left\{\bar{\psi}_{l_{m}}\right\}$, i.e.,

$\hat{s}_{l}\left(m \Delta_{t}\right)=\frac{1}{M} \alpha_{l_{M}}\left\langle\bar{\phi}_{l_{M}}, \bar{\psi}_{l_{m}}\right\rangle$

The function $\hat{x}_{l}(t)$ can be obtained from $\hat{s}_{l}(t)$ by

$\hat{x}_{l}(t)=\hat{s}_{l}(t)-A \sin (2 \pi f t)$.

Experimental results. Consider the original signal $x_{l}(t)$ that is periodic with period $T=1$ and possesses no harmonics above the fourth. Note that $x_{l}(t)$ has 8 zeros in the interval $[-0.5,0.5]$. However, only 6 of them are real zero crossings [see Fig. 4(a)]. We use the real-zero conversion method with $A=8$ and $f=5$. Because the number of zeros of the new bandlimited signal, $s_{l}(t)$, after conversion is equal to the dimension of the signal (10 real zeros), the zeros are sufficient to represent the signal $s_{l}(t)$ [see Fig. 4(b)]. The reconstructed signal depends on the accuracy of the estimate of the zero-crossing locations. The accuracy of the estimate of zero-crossing locations is determined by the number of sampling points used to estimate them. The total number of sampling points is $\left(N_{q} \times\right.$ the number of $\left.\Delta_{t}\right)$. Note that $N_{q}$ is the number of sampling points per $\Delta_{t}$ and the sampling rate in Fig. 5 is given by $\log _{2} N_{q}=p$ bits $/ \Delta_{t}$. We then find successive samples with opposite signs and, finally, linearly interpolate those samples to obtain the zero-crossing locations. Figure 5 shows the ESR of the estimator $s_{l}(t)$ depending on the accuracy of the estimate of the zero-crossing locations.

\subsubsection{Reconstruction of bandpass signals}

Consider a bandpass signal having support band $[-b,-a]$ and $[a, b]$ with bandwidth $\Omega=b-a$. This type of signal has dimension $2(b-a) / \omega_{0}$ but has $2 b / \omega_{0}$ zeros including real and/or complex zeros per period. Logan ${ }^{18}$ shows that a certain type of bandpass signal can be uniquely determined from its real zero crossings (within a multiplicative constant) even if it has complex zeros. He defined the number of real zero crossings of a bandpass signal in the following theorem.

Theorem (from Logan). Let $x_{p}(t)$ be a real-valued function in the band $[-b,-a] \cup[a, b], x_{p}(t) \neq 0$, and denote by $\beta(T)$ the number of sign changes of $x_{p}(t)$ in the interval $(0, T)$. Then

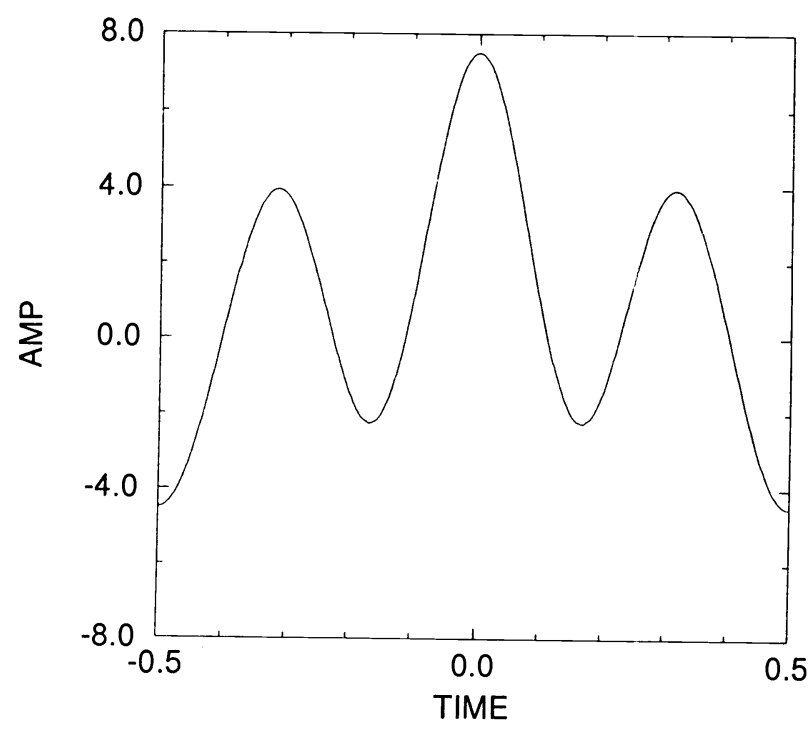

(a)

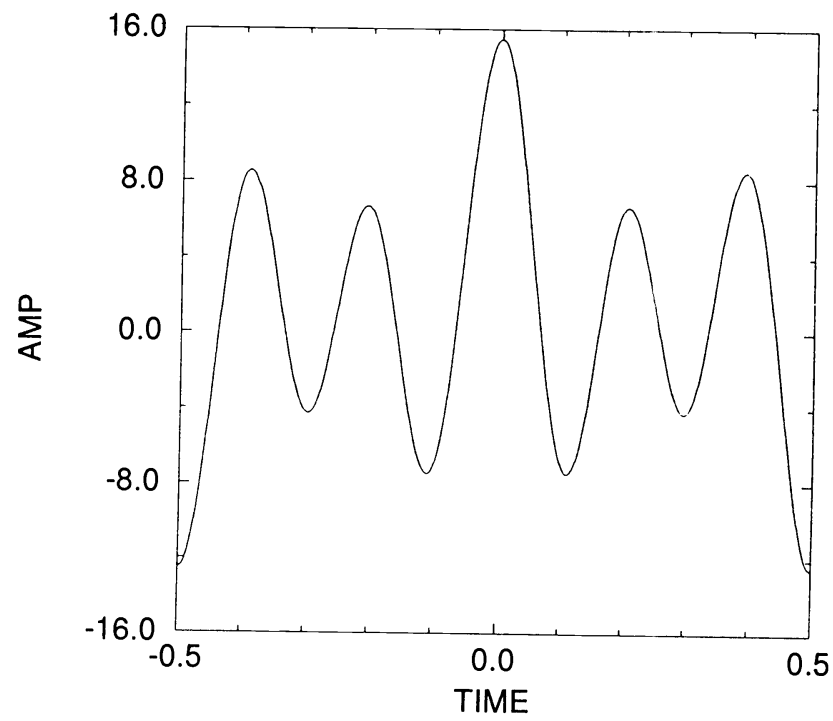

(b)

Fig. 4 (a) The original signal $x_{l}(t)$ and the reconstructed signal $\hat{x}_{l}(t)$, and (b) the converted signal $s_{l}(t)$ and the reconstructed signal $\hat{s}_{l}(t)$.

$\lim _{T \rightarrow \infty} \inf \frac{\beta(T)}{T} \geqslant \frac{a}{\pi}$

This theorem shows that the number of zeros of the signal that satisfies Logan's conditions exceeds the number of degrees of freedom of the signal where the average sampling rate exceeds the Nyquist rate.

Experimental results. The original test signal $x_{p}(t)$ is band-limited $[-16,-9] \cup[9,16]$. We need 16 values $(15$ zero crossings and 1 multiplicative constant) to apply our algorithm. However, the number of real zero crossings of this signal is 26 . We select only 15 zero crossings that give the minimum basis error. Figure 6 depicts the original signal with selected real zero crossings. Figure 7 shows the ESR of 


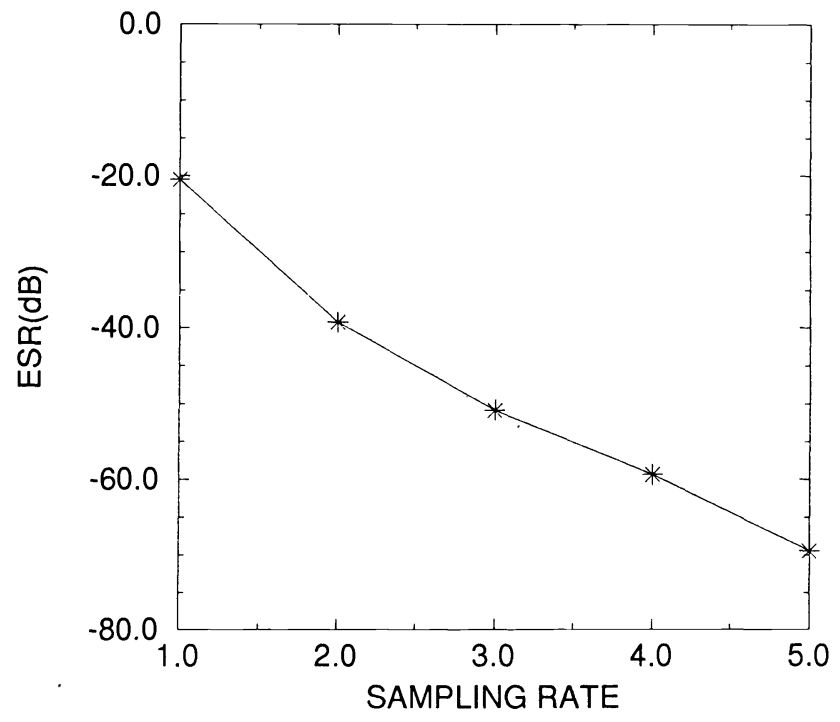

Fig. 5 The ESR of the estimator for the $s_{l}(t)$ depending on the accuracy of the zero locations.

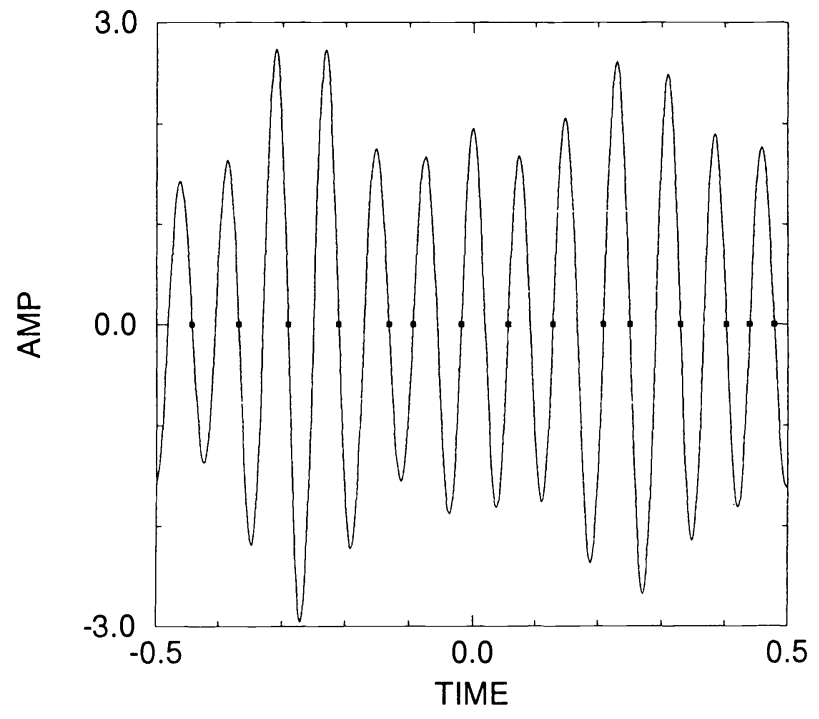

Fig. 6 The original bandpass signal $x_{p}(t)$ with zero crossings.

the estimator for $x_{p}(t)$ depending on the accuracy of the estimate of zero-crossing locations.

\subsection{Reconstruction of Images}

The nonorthogonal basis functions $\mathbf{W}_{\mathbf{I}_{\mathbf{L}}}$ that span the 2-D band-limited signal of nonuniform data are introduced in matrix form as follows:

$\mathbf{W}_{\mathbf{I}_{\mathrm{L}}}=\left[\begin{array}{ccccc}u_{x_{1}}^{-M} \mathbf{v}_{y_{1}} & u_{x_{1}}^{-M+1} \mathbf{v}_{y_{1}} \ldots & u_{x_{1}}^{M} \mathbf{v}_{y_{1}} \\ u_{x_{2}}^{-M} \mathbf{v}_{y_{1}} & u_{x_{2}}^{-M+1} \mathbf{v}_{y_{1}} \ldots & u_{x_{2}}^{M} \mathbf{v}_{y_{1}} \\ \vdots & \vdots & \ldots & \vdots \\ u_{x_{M}}^{-M} \mathbf{v}_{y_{N}} & u_{x_{M}}^{-M+1} \mathbf{v}_{y_{N}} \ldots & u_{x_{M}}^{M} \mathbf{v}_{y_{N}}\end{array}\right]$,

where $v_{y_{j}}^{N}=\exp \left(i N \omega_{0} y_{j}\right), u_{x_{j}}^{M}=\exp \left(i M \omega_{0} x_{j}\right)$ and $\mathbf{v}_{y_{j}}=\left[v_{y_{j}}^{-N}\right.$, $\left.v_{y_{j}}^{-N+1}, \ldots, v_{y_{j}}^{N}\right]$. Note that $K=M N$. We first construct orthonormal basis functions $\Gamma_{\mathbf{I}_{\mathrm{L}}}=\left[\bar{\gamma}_{I_{l 1}}, \bar{\gamma}_{I_{l 2}}, \ldots, \bar{\gamma}_{I_{l K}}\right]^{T}$ using the

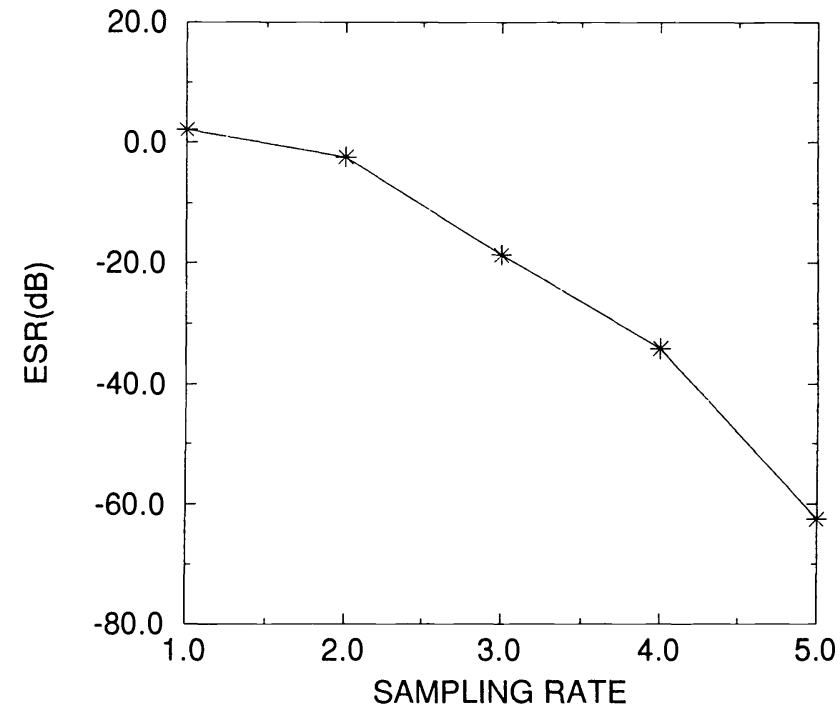

Fig. 7 The ESR of the $x_{p}(t)$ depending on the accuracy of the zero locations.

Gram-Schmidt procedure from $\mathbf{W}_{\mathbf{I}_{\mathrm{L}}}=\left[\bar{w}_{I_{l 1}}, \bar{w}_{l_{l 2}}, \ldots, \bar{w}_{l_{l K}}\right]^{T}$. The coefficients $\left\{\alpha_{I l}\right\}$ can be evaluated with the same procedure in the 1-D case. The first term in the numerator of Eq. (17) can be written

$$
\begin{aligned}
\left\langle\mathbf{F}_{\mathbf{I}_{\mathrm{L}}}, \bar{w}_{I_{l j}}\right\rangle= & \sum_{m=-M}^{M} \sum_{n=-N}^{N} F_{I_{l}}(m, n) \\
& \times \exp \left[-i\left(m \omega_{0} x_{k}+n \omega_{0} y_{l}\right)\right],
\end{aligned}
$$

where $\quad \mathrm{F}_{\mathrm{I}_{\mathrm{L}}}=\left[F_{I_{1}}(-M,-N), \quad F_{I_{I}}(-M+1,-N), \quad \ldots\right.$, $\left.F_{I_{I}}(M, N)\right]$. Since the nonuniform data and DFT spectrum can be expressed

$$
\left[\begin{array}{c}
f_{I_{l}}\left(x_{1}, y_{1}\right) \\
f_{I_{l}}\left(x_{2}, y_{1}\right) \\
\vdots \\
f_{I_{l}}\left(x_{M}, y_{N}\right)
\end{array}\right]=\frac{1}{K} \mathbf{W}_{\mathbf{I}_{\llcorner}} \cdot\left[\begin{array}{c}
F_{I_{l}}(-M,-N) \\
F_{I_{l}}(-M+1,-N) \\
\vdots \\
F_{I_{l}}(M, N)
\end{array}\right] \text {, }
$$

Eq. (58) can be written

$\left\langle\mathbf{F}_{\mathbf{I}_{\mathrm{L}}}, \bar{w}_{I_{l j}}\right\rangle=f_{I_{l}}\left(x_{k}, y_{l}\right) \cdot K$.

Hence,

$\alpha_{I_{l j}}=\frac{f_{I_{l}}\left(x_{k}, y_{l}\right) \cdot K-\sum_{k=1}^{j-1} \alpha_{I_{l k}}\left\langle\bar{\gamma}_{I_{l k}}, \bar{w}_{I_{l j}}\right\rangle}{\left\langle\bar{\gamma}_{I_{l j}}, \bar{w}_{I_{l j}}\right\rangle}$.

Finally, the desired image can be written as

$\hat{f}_{I_{l}}\left(m \Delta_{x}, n \Delta_{y}\right)=\frac{1}{K} \sum_{k=1}^{K} \alpha_{I_{l k}}\left\langle\bar{\gamma}_{I_{l k}}, \bar{\theta}_{I_{l m n}}\right\rangle$.

Experimental result. The 2-D function, which is bandlimited $u \in[-3,3]$ and $v \in[-3,3]$, is tested. We generate the nonuniform sampling set for the rate of deviation from the uniform sampling points and for the random sampling 
Table 3 The ESR for the 2-D gray-level image obtained from 30 sets of nonuniform samples.

\begin{tabular}{c|c}
\hline $\begin{array}{c}\text { DISTRIBUTION OF } \\
\text { SAMPLES }\end{array}$ & $\begin{array}{c}\text { ESR OF THE } \\
\text { SIGNAL (dB) }\end{array}$ \\
\hline $50 \%$ & -104.07 \\
\hline $100 \%$ & -92.51 \\
\hline $500 \%$ & -91.73 \\
\hline RANDOM & -89.23 \\
\hline
\end{tabular}

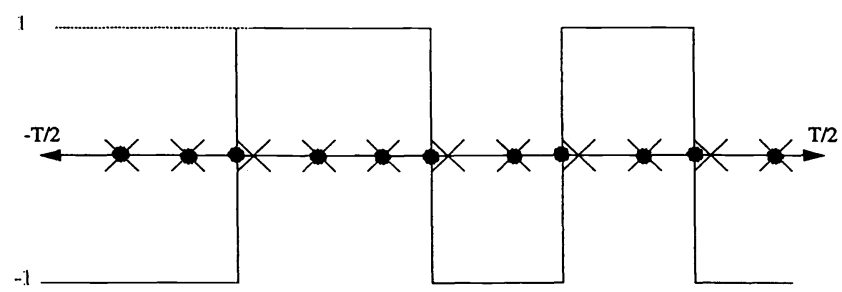

Fig. 8 The evenly spaced samples (" $x$ " marks) and the available zero-crossing points.

points in the intervals $x \in[-0.5,0.5]$ and $y \in[-0.5,0.5]$. Table 3 shows the average ESR of the estimator for the image obtained by 30 sets of nonuniform samples.

\subsection{Image Synthesis}

In this section we apply our algorithm to solve the problem of synthesis of a 2-D band-limited function with the prescribed level crossings. This problem arises when we want to generate a prescribed binary image at the output of a diffraction-limited imaging system with high-contrast recording. The imaging system we deal with is a linear bandlimited system followed by a hard-limiting point nonlinearity.

We first apply our algorithm to solve the problem of synthesis of a 1-D binary function. Let $f(x)$ be the 1-D binary function with the prescribed zero crossings. The marks " $x$ " in Fig. 8 denote the evenly spaced sampled points that satisfy the Nyquist rate for the given imaging system. The evenly spaced sampled points were replaced by the nearest real zero crossings. The dots in Fig. 8 denote the available points to synthesize a binary function.

Figure 9 depicts the binary function in the interval $[-0.5,0.5]$ with four prescribed zero crossings. Let the imaging system be band-limited $[-2,2$.]. The five samples are required to synthesize the binary function. The four evenly spaced samples are replaced by the nearest real zero crossings. The four real zero crossings and one evenly spaced sample are the available points to synthesize $f(x)$. The line function in Fig. 9(a) is the desired output function with 64 sampling points. The line plot in Fig. 9(b) shows the output function through the imaging system when the line function in Fig. 9(a) is fed into the imaging system. The output function is not the desired one. The dotted plot in Fig. 9(a) is the input function that is obtained by our algorithm. The desired function, which is the dotted one in Fig. 9(b), is obtained when the proposed input function is fed into the imaging system.

We here extend our algorithm to solve the problem of synthesis of a 2-D band-limited function. The process of

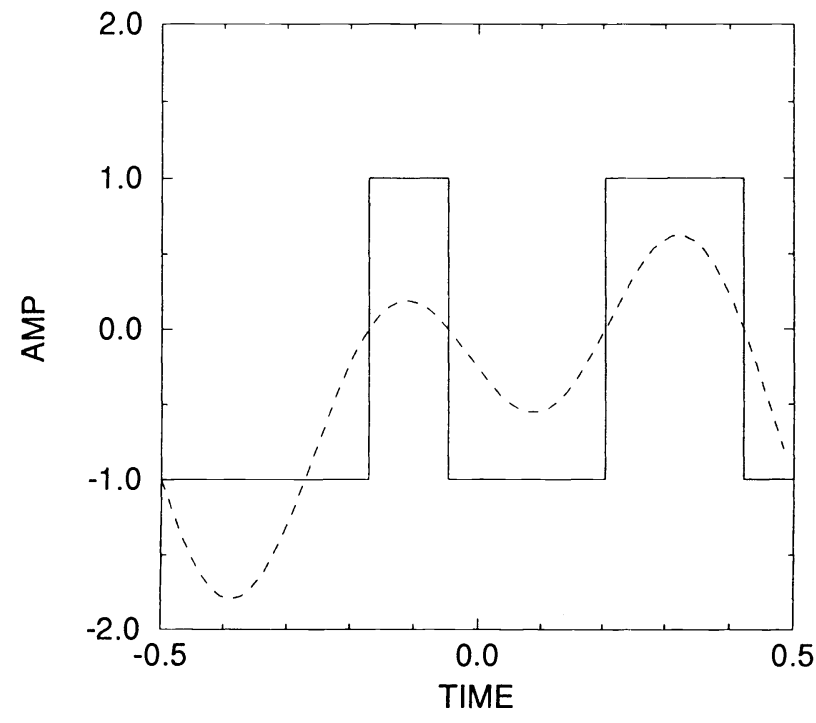

(a)

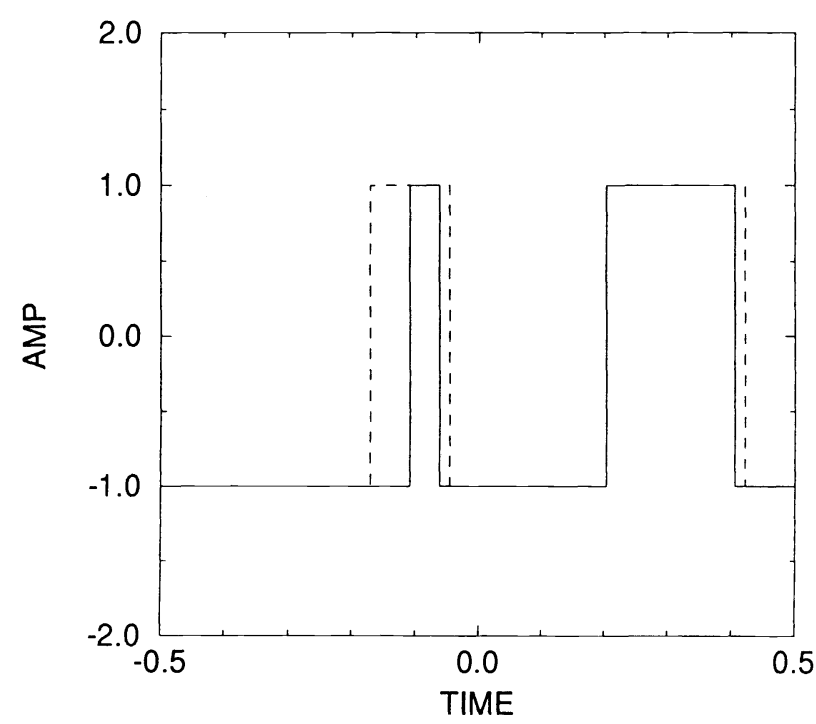

(b)

Fig. 9 (a) The desired output function (line) and the proposed bandlimited input function (dash); (b) the output function obtained from the input function (line) and the desired output function from the proposed input function.

replacing the level-crossing points is the same as it was for the 1-D case. The image in Fig. 10(a) is the desired output pattern. The number of pixels in the pattern is $32 \times 32$. The band region of the system is $[-5,5]$. Figure 10(b) shows the output pattern through the imaging system when the image shown in Fig. 10(a) is used as the input pattern. Since the output pattern in Fig. 10(b) is not the desired one, the direct approach is not appropriate. Figure 10(c) depicts the 2-D band-limited function that is obtained by our algorithm from the prescribed threshold crossings. When the image in Fig. 10 (c) is fed into the image system, the desired output pattern is generated as shown in Fig. 10(d).

\section{Conclusion}

We studied a deterministic signal subspace process via the Gram-Schmidt orthogonalization procedure to reconstruct 


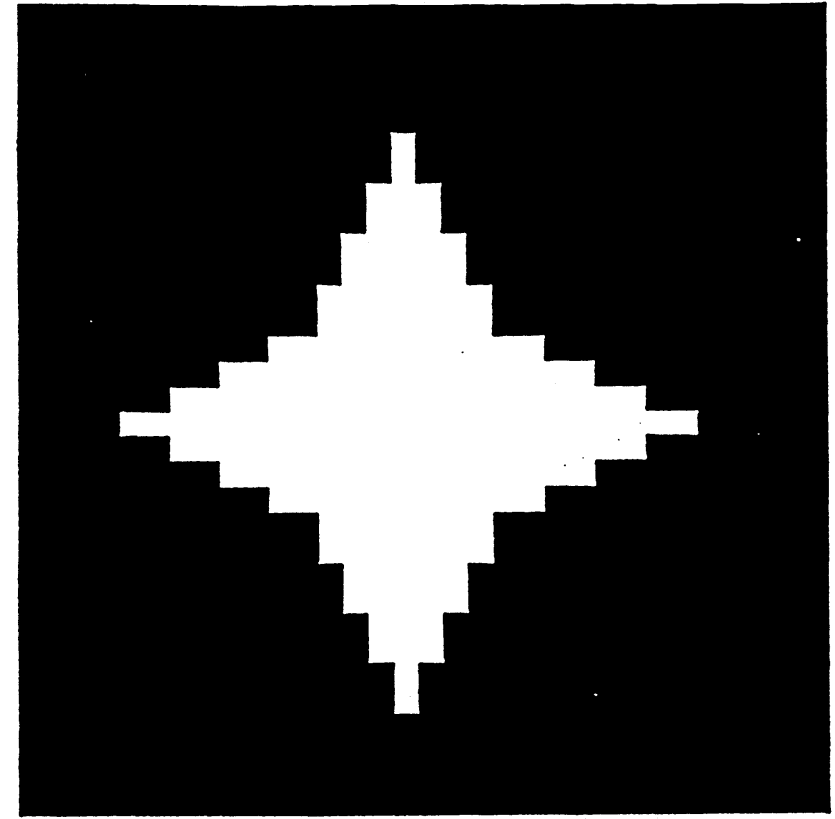

(a)

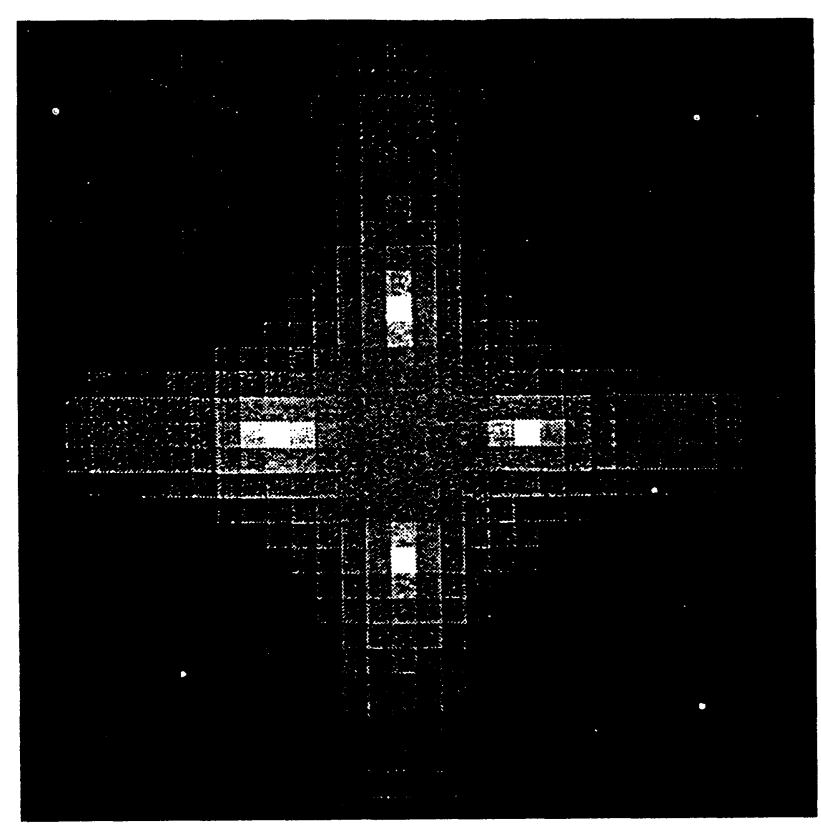

(c)

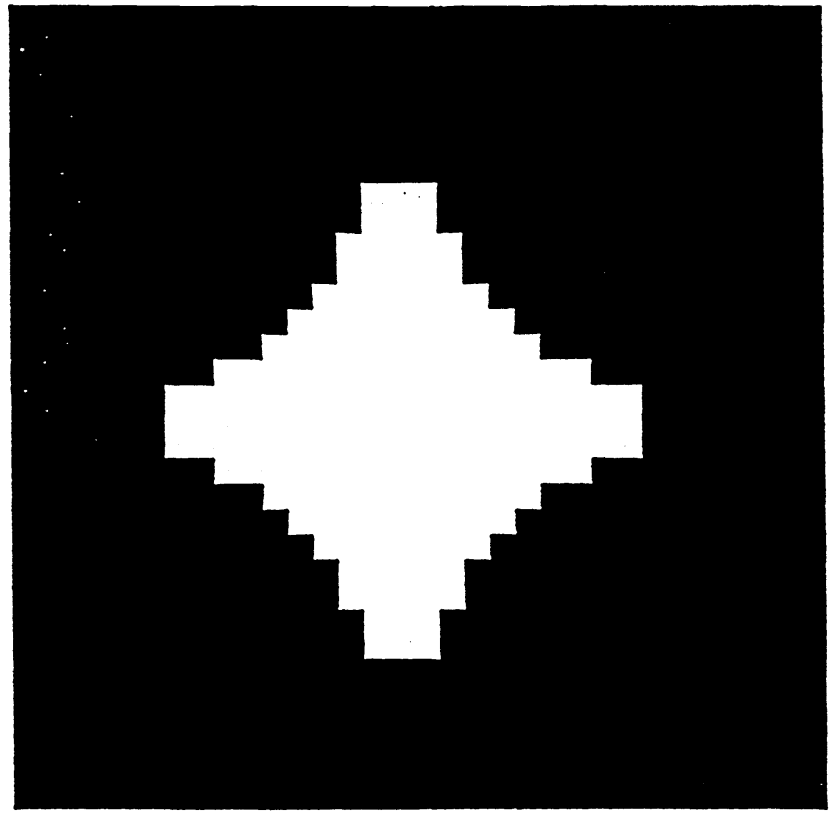

(b)

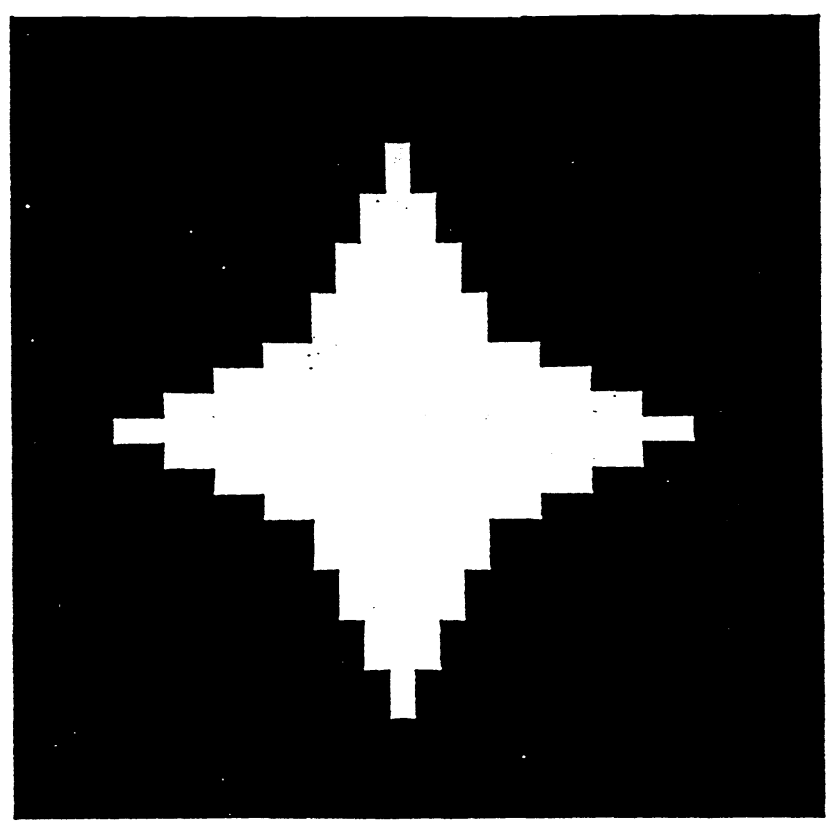

(d)

Fig. 10 (a) The desired output pattern used as input pattern; (b) the output pattern from (a); (c) the proposed input pattern; and (d) the desired output pattern.

functions from their unevenly spaced sampled data and zero/ threshold crossings. We also showed the numerical results to determine the completeness of the basis functions. We apply our algorithm to reconstruct functions from their unevenly spaced sampled data and zero crossings and apply it to solve the problem of synthesis of a 2-D band-limited function with the prescribed level crossings. The reconstructed signal gives the MMSE estimate of the original signal. This procedure gives error-free reconstruction provided that the nonorthogonal basis functions that span the signal subspace of the nonuniform data form a complete set in the signal subspace of the original band-limited signal.

\section{Appendix: Reconstruction by the Matrix Inversion}

In this section we estimate a signal by the matrix inversion. We represent $\hat{\mathbf{F}}$ by the coefficients $\left\{c_{k} ; i=1,2, \ldots, M\right\}$ with respect to the basis $\left\{\bar{u}_{k} ; k=1,2, \ldots, M\right\}$, i.e., 
$\hat{\mathbf{F}}=\sum_{k=1}^{M} c_{k} \bar{u}_{k}$

By projection theory we can write

$\left\langle\mathbf{F}=\hat{\mathbf{F}}, \bar{u}_{j}\right\rangle=0 \quad$ for $j=1,2, \ldots, M$.

By linearity of the inner product, Eq. (64) can be rewritten as

$\left\langle\mathbf{F}, \bar{u}_{j}\right\rangle=\sum_{k=1}^{M} c_{k}\left\langle\bar{u}_{k}, \bar{u}_{j}\right\rangle \quad$ for $j=1,2, \ldots, N$.

Equation (65) can be written in the matrix form using the same procedure utilized in Sec. 3, i.e.,

$\left[\begin{array}{c}f\left(t_{1}\right) \\ f\left(t_{2}\right) \\ \vdots \\ f\left(t_{M}\right)\end{array}\right]=\frac{1}{M} \mathbf{V} \cdot\left[\begin{array}{c}c_{1} \\ c_{2} \\ \vdots \\ c_{M}\end{array}\right]$

where $\mathbf{V}$ is given by

$\mathbf{v}=\left[\begin{array}{cccc}\left\langle\bar{u}_{1}, \bar{u}_{1}\right\rangle & \left\langle\bar{u}_{2}, \bar{u}_{1}\right\rangle & \ldots & \left\langle\bar{u}_{M}, \bar{u}_{1}\right\rangle \\ \left\langle\bar{u}_{1}, \bar{u}_{2}\right\rangle & \left\langle\bar{u}_{2}, \bar{u}_{2}\right\rangle & \ldots & \left\langle\bar{u}_{M}, \bar{u}_{2}\right\rangle \\ \vdots & \vdots & \ldots & \vdots \\ \left\langle\bar{u}_{1}, \bar{u}_{M}\right\rangle & \left\langle\bar{u}_{2}, \bar{u}_{M}\right\rangle & \ldots & \left\langle\bar{u}_{M}, \bar{u}_{M}\right\rangle\end{array}\right]$

The elements of the matrix $\mathbf{V}$, which is the inner product of the $k$ 'th basis $u_{k}$ and $j$ 'th basis $u_{j}$, can be written as follows:

$$
\begin{aligned}
\left\langle u_{k}, u_{j}\right\rangle & =\sum_{k=-N}^{N} \exp \left[i k \omega_{0}\left(t_{k}-t_{j}\right)\right] \\
& =\frac{\sin \frac{(2 N+1)}{2} \omega_{0}\left(t_{k}-t_{j}\right)}{\sin \frac{1}{2} \omega_{0}\left(t_{k}-t_{j}\right)} \\
& =p\left(t_{k}-t_{j}\right) .
\end{aligned}
$$

Thus, the coefficients $\left\{c_{k}\right\}$ can be expressed as follows:

$$
\left[\begin{array}{c}
c_{1} \\
c_{2} \\
\vdots \\
c_{M}
\end{array}\right]=\frac{1}{M} \cdot\left[\begin{array}{cccc}
M & p\left(t_{2}-t_{1}\right) & \ldots & p\left(t_{M}-t_{1}\right) \\
p\left(t_{1}-t_{2}\right) & M & \ldots & p\left(t_{M}-t_{2}\right) \\
\vdots & \vdots & \ldots & \vdots \\
p\left(t_{1}-t_{M}\right) & p\left(t_{2}-t_{M}\right) & \ldots & M
\end{array}\right]^{-1} \cdot\left[\begin{array}{c}
f\left(t_{1}\right) \\
f\left(t_{2}\right) \\
\vdots \\
f\left(t_{M}\right)
\end{array}\right] .
$$

We used an IMSL/LIB subroutine (DLINRG) to determine the inverse matrix, which is used with real double precision data. Hence, we can reconstruct a signal using the following expression:

$$
\hat{f}\left(m \Delta_{t}\right)=\sum_{k=1}^{M} c_{k}\left\langle\bar{u}_{k}, \bar{\psi}_{m}\right\rangle \text {. }
$$

\section{References}

1. A. J. Jerri, "The Shannon sampling theorem-Its various extensions and applications: A tutorial review,'’ Proc. IEEE 65, 1565-1596 (Nov. 1977).

2. F. Marvasiti, A Unified Approach to Zero-Crossings and Nonuniform Sampling of Single and Multidimensional Signals and Systems, Nonuniform, Oak Park, IL (1987).

3. H. Stark, Image Recovery Theory and Applications, Academic Press, New York (1988).

4. M. Soumekh, "Band-limited interpolation from unevenly spaced sampled data," IEEE Trans. Acoust. Speech Signal Proc. ASSP-36, 110-122 (Jan. 1988).

5. M. Soumekh, "Reconstruction and sampling constraints for spiral data," IEEE Trans. Acoust. Speech Signal Proc. 37, 882-891 (1989).

6. J. J. Clark, M. Palmer, and P. Lawrence, "A transformation method for the reconstruction of functions from nonuniformly spaced samples,' IEEE Trans. Acoust. Speech Signal Proc. ASSP-33, 1151-1165 (Oct. 1985).

7. A. G. Requicha, "The zeros of entire functions: theory and engineering applications," Proc. IEEE 68(3), 308-326 (Mar. 1980).

8. S. J. Haavik, "The conversion of the zeros of noise," MSc Thesis, University of Rochester, NY (1966).

9. S. Kay and R. Sudhaker, "A zero crossing-based spectrum analyzer," IEEE Trans. Acoust. Speech Signal Proc. ASSP-34, 96-104 (Feb. 1986).

10. K. M. Nashold and B. E. A. Saleh, "Synthesis of two-dimensional binary images through band-limited systems: a slicing method," IEEE Trans. Acoust. Speech Signal Proc. ASSP-37, 1271-1279 (Aug. 1989).

11. S. I. Sayegh, Y. L. Kok, and J. H. Hong, "An algorithm to find twodimensional signals with specified zero crossings," IEEE Trans. Acoust. Speech Signal Proc. ASSP-35, 107-111 (Jan. 1987).

12. S. I. Sayegh, B. E. A. Saleh, and K. M. Nashold, "Image design: generation of a prescribed image through a diffraction-limited system with high-contrast recording," IEEE Trans. Acoust. Speech Signal Proc. ASSP-33, 460-465 (Apr. 1985).

13. F. J. Beutler, "Error-free recovery of signals from irregularly spaced samples," SIAM Rev. 8, 328-335 (July 1966).

14. J. R. Higgins, "A sampling theorem for irregularly spaced sample points,' IEEE Trans. Info. Theory IT-21, 621-622 (Sep. 1976).

15. D. S. Chen and J. P. Allebach, "Analysis of error in reconstruction of two-dimensional signals from irregularly spaced samples," IEEE Trans. Acoust. Speech Signal Proc. ASSP-35, 173-180 (Feb. 1987).

16. J. L. Yen, 'On nonuniform sampling of bandlimited signals,' IRE Trans. Circuit Theory CT-3, 251-257 (Dec. 1956).

17. J. L. Brown, Jr., "On uniform sampling of amplitude modulated signals,' IEEE Trans. Aerospace Electron. Syst. AES-19, 633-635 (July 1983).

18. B. F. Logan, Jr., "Information in the zero crossings of bandpass signals," The Bell Syst. Tech. J. 56, 487-510 (Apr. 1977).

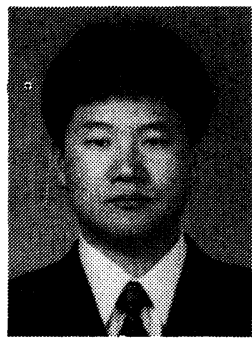

Yongwan Park received the BS and MS degrees in electronics from Kyungpook National University, Taegu, Korea, in 1982 and 1984, respectively, and MS and PhD degrees in electrical and computer engineering from State University of New York (SUNY) at Buffalo in 1989 and 1992, respectively. From 1987 to 1992 , he was a research and teaching assistant at SUNY. From October 1992 to December 1993, he worked as a research fellow at the California Institute of Technology, Pasadena, California. In 1994, he joined the Central Research Center of Korea Mobile Telecom as the director of system technology in the CDMA Task Force. His research interests are digital signal/image processing, mobile telecommunication including digital cellular systems, and PCS.

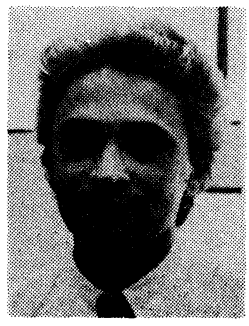

Mehrdad Soumekh received the BEE and $\mathrm{PhD}$ degrees from the University of Minnesota. He is currently an associate professor of electrical engineering at the State University of New York at Buffalo. His research interests are in the areas of signal processing and inverse scattering. $\mathrm{He}$ is the author of the book Fourier Array Imaging (Prentice-Hall, 1994). 